\title{
ERRATUM, VOLUME 70
}

Melvin Henriksen and J. R. Isbell, Averages of continuous functions on countable spaces, pp. 287-290.

Theorem 1 is essentially a special case of Theorem 3, p. 209 of A. D. Alexandroff's paper Additive set-functions in abstract spaces, Mat. Sbornik (N.S.) 13 (55) (1943), 169-238. The authors are indebted to E. Granirer for pointing this out. 